\title{
Design, Modelling and Simulation of a Capacitive size-discriminating Particulate Matter Sensor for personal air quality monitoring.
}

\author{
Pelumi W. Oluwasanya, Member, IEEE, Girish Rughoobur, Member, IEEE, and Luigi G. Occhipinti
}

\begin{abstract}
We applied the well-established thermophoretic effect to air quality monitoring. We developed a novel method for particulate matter distribution analysis in an in-flow capacitive detection device. The proposed Multiphysics model combines fluid dynamics of particulate matter influenced by thermophoresis with electric field variations in the active volume space of a charged coplanar interdigitated electrodes. The model allows to anticipate the effect of thermophoresis in separating particles of PM10 and PM2.5 size ranges into different streams from a single particleentrained flow and provides an estimated value of sensitivity for capacitive PM detection. The model is described through the Finite Element Method from the main equations to the simulation run using COMSOL Multiphysics and validated by comparing the results with literature. We obtained high sensor sensitivity of up to $0.48 \mathrm{zF} /$ particle as far as $\mathbf{1 8} \boldsymbol{\mu \mathrm { m }}$ from coplanar electrode surface using the Computational Fluid Dynamics and Heat Transfer, Electrostatics and Particle tracing modules. We compare results of the simulations for different particle positions, electrode width and inter-electrode spacing, then we use the results to identify optimal design parameters for a novel architecture of a PM detection system with high sensitivity down to PM2.5 single particles and embedded particle size discrimination by using 10 $\mu \mathrm{m}$ electrode width and $1 \mu \mathrm{m}$ inter-electrode spacing.
\end{abstract}

Index Terms-Capacitive PM2.5 sensor; Thermophoresis; Particulate Matter Detection; Air quality monitoring, Particle size discrimination; Finite Element Method.

\section{INTRODUCTION}

$\mathrm{E}$ NSURING A safe and clean air and environment is a non-negotiable prerequisite to promoting global health, well-being and reducing air pollution-related mortality. Harmful substances released into the atmosphere by human, natural and industrial processes, especially particles, persist in indoor environments causing higher mortality than outdoor exposures [1]. Particulate Matter (PM) is seen as a cause for

Submitted 6th June 2019. This work was supported in part by InnovateUK (GraphClean G.A. No. 71476-481865 and MP-SENS 73047-501268), EPSRC Sensors CDT (G.A. No. EP/KO3099X/1), EPSRC CIMLAE (G.A. No. EP/LO15889/1) and the Presidential Special Scholarship for Innovation and Development (PRESSID) managed by the National Universities Commission (NUC) and funded by the Petroleum Technology Development Fund (PTDF).

Pelumi W. Oluwasanya is a $\mathrm{PhD}$ candidate in Electrical Engineering Division, University of Cambridge Department of Engineering, 9 JJ Thomson Avenue, Cambridge CB3 OFA. tens of thousands of premature deaths annually in the United Kingdom alone. Therefore, an accurate estimation of exposure to particulate matter is essential. Further, epidemiological studies have shown higher correlation between PM exposure and health problems, mostly respiratory, cardiovascular [2][7], hospital admissions and emergency room visits [8]-[12] than with other outdoor pollutants [13].

Gravimetric sensors are very accurate in measuring PM mass concentration obtained from the difference in mass before and after PM deposition. They are mostly bulky and are most commonly used in air quality monitoring sensor networks. Beta attenuation measurement is one of such used to analyze particles after collection on filters. The amount of beta radiation absorbed by these solid particles is a function of its mass, thus PM mass concentration can be obtained from the measurement. Beta Attenuation Monitoring (BAM) [14], [15] is quite popular and still in use because it is an online method and when coupled with cyclones, or some other size discrimination method, it can detect and monitor particles of different sizes. Another example is the Tapered Element Oscillating Microbalance (TEOM) [16].

However, given the sparseness of the air quality monitoring networks ( 300 sites in the UK), the resolution of the data obtained is low. Whereas accurate estimate of the variation in air pollution measurements in spatial and temporal dimensions when fully captured, will provide deeper understanding and may inform tailor-made and more effective exposure mitigation approaches [17]-[19]. This has proven difficult to achieve with many commercially available (mostly light-based) PM sensing devices without compromising accuracy [20], [21].

Other portable techniques include acoustic-based PM sensors which use the shift in the resonant frequency of a resonating sensor element when PM is deposited on it to determine the PM mass concentration using the Sauerbrey equation, and

Girish Rughoobur is a Postdoctoral researcher at Massachusetts Institute of Technology, Department of Electrical Engineering and Computer Science, Cambridge, United States.

Luigi G. Occhipinti is Director of Research at Department of Engineering, University of Cambridge, 9 JJ Thomson Avenue, Cambridge CB3 0FA. Phone: +447548 323462, Email: luigi.occhipinti@eng.cam.ac.uk (corresponding author) 
impedance-based methods. These usually target a single size range which is usually possible when particles outside the target range are filtered away by suitable means. Filtering away particles of specific sizes from the sample volume, however, means that the statistics of particle distribution in the sample volume of air is affected by the actual measurement. It is possible to obtain a simultaneous, statistically correct, distribution of the particles entrained in the sampled volume of air flow that fall in the PM2.5 and in the PM10 ranges without altering the overall distribution of the smaller and larger particles present in the sample volume via capacitive method coupled with thermophoresis. Capacitance-based PM sensors [22], [23] are getting increasing research attention because they can be miniaturized, can be easily integrated with an effective particle discrimination technique and can have simpler detection electronics with off-the-shelf capacitive readouts such as the MS3110 from Irvine Sensors.

This paper studies the motion of sampled particle-entrained airflow in a portable model under the influence of thermophoresis, which successfully separated particles of PM10 and PM2.5 size ranges. This was achieved by coupling the Computational Fluid Dynamics, Heat Transfer as well as Particle tracing modules of COMSOL Multiphysics $₫$ v.3a, and v5.4. Detection was verified using capacitive method [23] using a differential electrode pair at a single particle detection level thus pushing the limits of the resolution of currently available PM sensor systems and readout electronics. This is a proposed improvement from related works such as Sun et al., [22], [23] which reported data obtained after deposition of incremental mass of particles. For that, the mass of both the sensor element and particles must be monitored simultaneously during the experiment at a constant flow rate. In fact, to make the system work with high resolution and time accuracy, the deposition rate should remain constant over the duration of the entire measurement experiment, which is very difficult to achieve in practice. In our approach the sensing elements are arranged to intercept the flow and the proposed model remains valid without restrictions related to the time fluctuation of the number of particles in a given size range that fall in the active sensing area.

The motion of particles across gradients, whether thermal, electric, or concentration is a well-established phenomenon and has applications already in several fields. Thermophoresis, the effect causing particles to respond differently to a thermal gradient, has been applied to the study of proteins [24], [25], the growth in a chemical vapor deposition reactor [26], drug delivery [27], and the study of fluid motion at a heated boundary for different flow conditions [28], [29]. While this established concept has been proven to be effective in these situations, its application to air quality monitoring has not been explored for particle size discrimination. Fundamentally, thermophoresis is a more effective particle discrimination technique than most other inertia-based separation techniques such as impactors and virtual impactors which usually have a size distribution in the major and minor flows. Filters can also get clogged, blocking off particles of the desired size. These preventable problems have led to the relative inaccuracies, and unit-to-unit deviations in some portable devices incorporating these techniques.

This paper focuses on the modelling of the effects of thermophoresis on particulate matter of PM10 and PM2.5 size ranges entrained in an airflow and how this provides a novel technique for monitoring particulate matter with possibility to further miniaturize and use in personal exposure studies. We designed our model in a similar way to Fotiadis and Jensen [26] where thermophoresis was used to create a particle-free region to remove impurities from the growth region using laminar flow regime, and validate using comparison to this, and capacitive measurement trends in Carminati et al [23].

\section{SENSOR MODELLING AND DESIGN}

\section{A. Model Assumptions}

Assumptions made in this work include laminar flow (to simplify the analysis in the two-dimensional model presented without significantly impacting the results), continuous fluid condition, low Reynold's number, low Knudsen number, infrequent particle-particle collisions, short particle settling velocity, non-isothermal conditions and linear contribution of finite infinitesimal volume elements in the inter-electrode gap to the total permittivity of inter-electrode space of the coplanar capacitors. Others include convective heat transfer flux, and the heat transfer coefficient of 10 . The analysis does not consider gas-aerosol conversion processes, and coagulation within the sensing chamber. Hence, merged particles present as single particle of a larger diameter to the sensor as this would also be the case from an exposure point of view. The simulations reported herein to validate our model have considered particles having different size ranges but the same average dielectric properties. While atmospheric particles may have different chemical and physical properties deviating from the ones adopted in the simulations the model is sufficiently accurate to be applied to indoor environments where the particle type range is narrower, for example.

\section{B. Theory}

Fluid flow in the laminar non-isothermal conditions in the sensing chamber was described by Fotiadis and Jensen [26]. The equations are presented below:

$$
\begin{aligned}
& \rho(\boldsymbol{u} \cdot \nabla) \boldsymbol{u}=\nabla \cdot\left[\left(-p I+\mu\left(\nabla \boldsymbol{u}+(\nabla \boldsymbol{u})^{T}\right)-\frac{2}{3} \mu(\nabla \cdot\right.\right. \\
& \boldsymbol{u}) I)]+\boldsymbol{F}_{\boldsymbol{F}} \\
& \nabla \cdot(\rho \boldsymbol{u})=0 \\
& \rho C_{F} u \cdot \nabla T+\nabla \cdot q=Q+Q_{p}+Q_{v d} \\
& \quad \boldsymbol{F}=m \frac{d \boldsymbol{u}_{p}}{d t}=\boldsymbol{F}_{D}+\boldsymbol{F}_{T}+\boldsymbol{F}_{g}
\end{aligned}
$$

Where $\boldsymbol{u}$ is the flow velocity, $I$ is an identity matrix, $m$ is the mass of the particle, $\boldsymbol{u}_{\boldsymbol{p}}$ is the velocity of the particle, $q$ is the heat flux, $Q, Q_{p}$, and $Q_{v d}$ represent the heat source, pressure work, and viscous dissipation respectively, the thermophoretic 
force is $\boldsymbol{F}_{T}, \nabla T$ is a uniform temperature gradient, $T$ is absolute temperature, $\rho$ is the density and $\mu$ is the coefficient of shear viscosity.

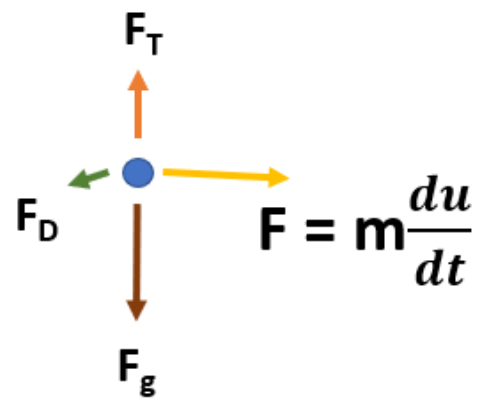

Fig. 1 - Forces acting on each particle.

Equations (1), (2) and (3) govern the fluid dynamics in a laminar non-isothermal flow regime. Equation (1) is the conservation of momentum equation. $\boldsymbol{F}_{\boldsymbol{F}}$ is the resultant force. Equation (2) is the conservation of mass equation. Equation (3) is the energy balance equation, and accounts for heat transfers via convection, conduction and radiation, while equation (4) is the particle's equation of motion with the forces shown in Fig. 1 , and it accounts for the drag force $\boldsymbol{F}_{D}$, thermophoretic force $\boldsymbol{F}_{T}$, and gravity $\boldsymbol{F}_{g}$. These forces are defined in the appendix.

The capacitance $(C)$, and electric field $(E)$, of the coplanar capacitors are also obtained by finite element analysis modelling from equations (A5) - (A7) reported in the appendix [30]. The increase in the relative permittivity of the space between coplanar capacitor electrodes when a particle enters their active volume space depends on the ratio between the particle aerodynamic volume and the volume of the active volume space between the charged electrodes and on the relative permittivity of the particle. The total permittivity of the region, $\varepsilon_{T}$ including the particle, is given by:

$$
\varepsilon_{T}=\left(m \varepsilon_{r}+(1-m)\right) \frac{v_{i}}{V} \varepsilon_{0}
$$

Where $m$ is the number of particles of type $i, V_{i}$ is the volume of a single particle, $\varepsilon_{r}$ is the relative permittivity and $V$ is the total volume of the inter-electrode gap. Finite element modelling (FEM), and analysis thus comes in handy for proper treatment of the resulting mathematical problem and is used here to obtain the fluid flow velocities, pressure, temperature variations, particle trajectories, electric field and hence the capacitance of coplanar electrode plates in the sensing chamber.

\section{Finite element method and model design}

Fig. 2 shows the non-isothermal flow COMSOL model Computer Aided Design (CAD). Bezier polygons were used to impose boundary conditions such as walls for the sensors, a heating surface for the heater, and the inlet/outlet. The walls were modelled as non-slip boundaries. Air was selected as the material of the housing domain. The inlet boundary condition was set to mass flow and flow rate of $100 \mathrm{sccm}$ (standard cubic centimeter) was used, where the non-isothermal flow study was chosen to be stationary.

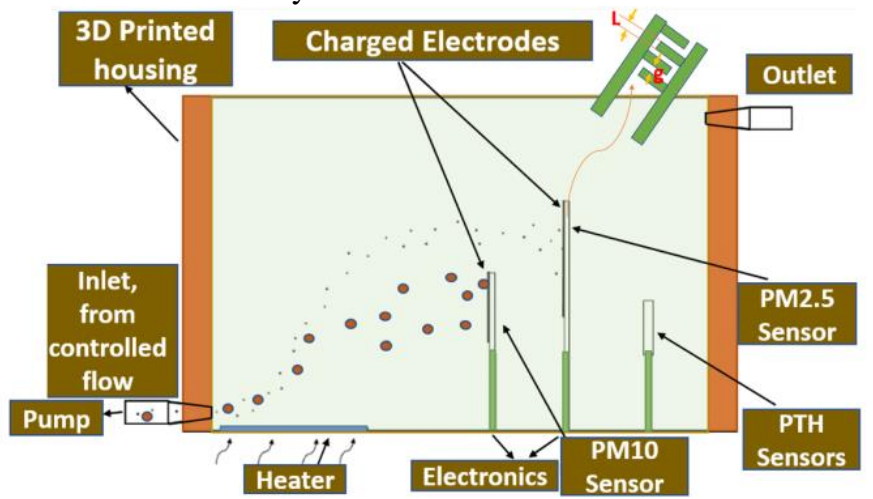

Fig. 2 - PM sensor and housing CAD design and model illustrating PM2.5 and PM10 discrimination via thermophoresis.

The outlet boundary was set to be at atmospheric pressure. The physical dimension of the housing was $50 \mathrm{~mm} \times 20 \mathrm{~mm} \times 14$ $\mathrm{mm}$. Heat flux within the chamber is chosen as convective and the heat transfer coefficient set to 10. Material selection proceeded as follows. The substrate, electrodes and particles were chosen to be glass, gold and silica respectively.

In the finite element model simulation, laminar flow was coupled with heat transfer in the study involving thermophoresis using the COMSOL Multiphysics interface non-isothermal laminar flow. The non-isothermal interface accounts for varying material properties due to the coupling such as the density during solution. Where the density of the material finite element is given as a function of the temperature and the reference value. The results of the single-phase nonisothermal analysis which include the temperature, velocity and pressure profiles were then coupled with particle tracing to obtain particle trajectories for the flow conditions. For simplicity and shorter computation time, thermophoresis was simulated only in two dimensions (see Fig. 2). The forces the particles experience include drag, and gravity. Brownian force was found to not have a significant effect on the particle flow, same as particle-particle collisions, as expected. Collision between the particles and the inner walls of the housing was set to bounce, the collision between the particles and the outlet wall was set to freeze. The density of the particle was set to 2200 $\mathrm{kg} / \mathrm{m}_{3}$ (COMSOL default value for silica). The actual sensing layers were implemented as walls placed at different heights which represent the areas where the particles of the target sizes are expected to be intercepted. The sensing surface was set to stick to the particles upon interception [31]. This may be enhanced in the prototype by coating the electrode surface with a thin layer of Poly Vinyl Alcohol (PVA). The particle tracing study involves the simultaneous release of particles of PM10 and PM2.5 sizes through the inlet into the sensing chamber. In the electrostatics simulation, sweep function was applied across variables such as particle trajectory coordinates and dielectric constants. For each sequential run of the electrostatic parametric sweep analysis, a particle is placed in a given position, displacing air corresponding to its own volume, the model solved, and capacitance obtained. At the next run the particle is then moved to another position until this is done for 


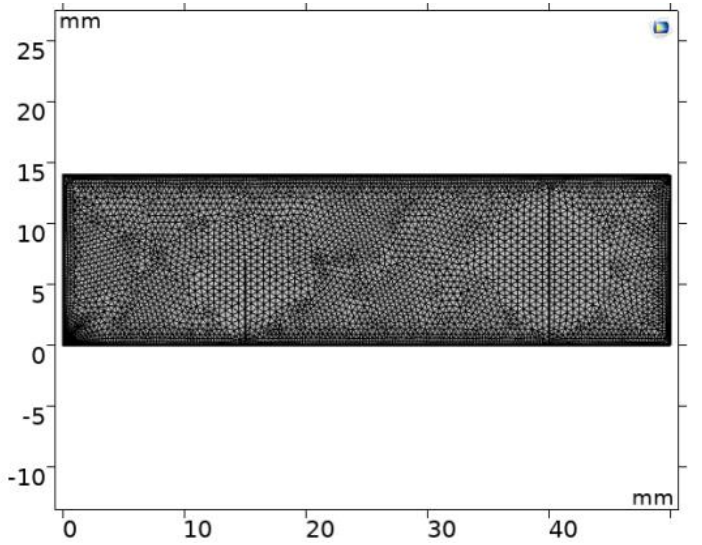

Fig. 3 - Finite element modelling mesh for solving the model.

all possible positions of the particle in the domain. The electrostatics simulation presents opportunity to optimize the sensor to attain the highest detection limit, by varying the critical parameters and comparing the results.

The inter-electrode space, the electrode width, the dielectric constant of the particle, and the height of the particle above the sensor surface can all be adjusted in the model to assess the feasibility of such a device. For example, for manufacturing purposes, it may be easier to make a sensor with $5 \mu \mathrm{m}$ interelectrode spacing than one with a $1 \mu \mathrm{m}$ spacing, and this should be possible without a significant reduction in sensor performance.

In all analysis, the mesh size was set to fine to minimize possible errors. This generated 362357 domain elements, 50044 boundary elements, and 5186 edge elements, shown in Fig. 3. Initial conditions were specified as follows: at $t=0, x=0$, $y=0.1 \mathrm{~mm}, \boldsymbol{u}=0.2 \mathrm{~m} / \mathrm{s}, \boldsymbol{u}_{P 0}=0$. A full description of the solution process through finite element modelling is presented in Fotiadis and Jensen [26].

\section{FEM tool challenges}

The main challenge with the finite element analysis tool is its inability to couple both non-isothermal fluid flow analysis and particle tracing with electrostatics. This implies that the obtained trajectory geometries of particles as they flow through the fluid will need to be either manually coupled for electrostatics model sweep at the desired points listed or simply simulating the electrostatics at all points in the domains of interest. The latter was used. Only PM2.5 was considered for the electrostatics simulation to validate the signal and sensor.

\section{RESULTS}

\section{A. Velocity profile}

Non-isothermal flow study results show the elliptic velocity profile for the fluid flow through the chamber. The result is consistent with expectations for non-isothermal laminar flow. The effect of thermophoresis, which is responsible for the upward tilt of the elliptic profile head over the volume space on top of the heater region, is shown in Fig. 4 (zoomed inset). This effect is clearly different from laminar flow streamline due to the pressure gradient across the chamber.

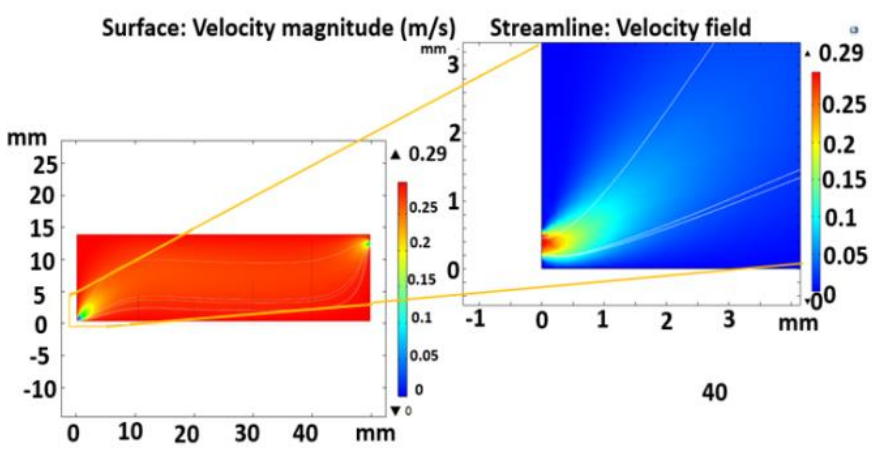

Fig. 4 - Velocity profile of the fluid flow with an enlargement showing the influence of thermophoresis on the bending of the flow trajectory away from the heater surface as it enters the heated region.

The results show that with a heater temperature of only $100{ }^{\circ} \mathrm{C}$, the effect of thermophoresis is clearly visible. The flow surface coloration gets darker as it approaches the walls of the housing indicating zero flow at the walls because of the non-slip boundary condition.

\section{B. Temperature profile}

The temperature profile is shown in Fig. 5. It shows the effect of the carrier fluid flow inertia on the temperature profile with the apparent draw-out of the elliptic shape to the right, compared with one with a stationary carrier medium. It also shows the thermal gradient across the chamber above the heater. This temperature profile is better suitable for understanding what Fotiadis and Jensen [26] described as the particle-free region (due to the thermophoretic effect) which has a similar profile over the heater region to the temperature gradient. The greater the temperature gradient, the greater the thermophoretic force, and thus the larger this region.

Turbulence may set in as the temperature increases beyond a certain limit leading to wall losses. This limit is a function of the thermal gradient, the housing geometrical dimensions, flow velocity and the density of the particle. The turbulence may also be caused by particle-wall collisions and the boundary condition imposed - elastic collision.

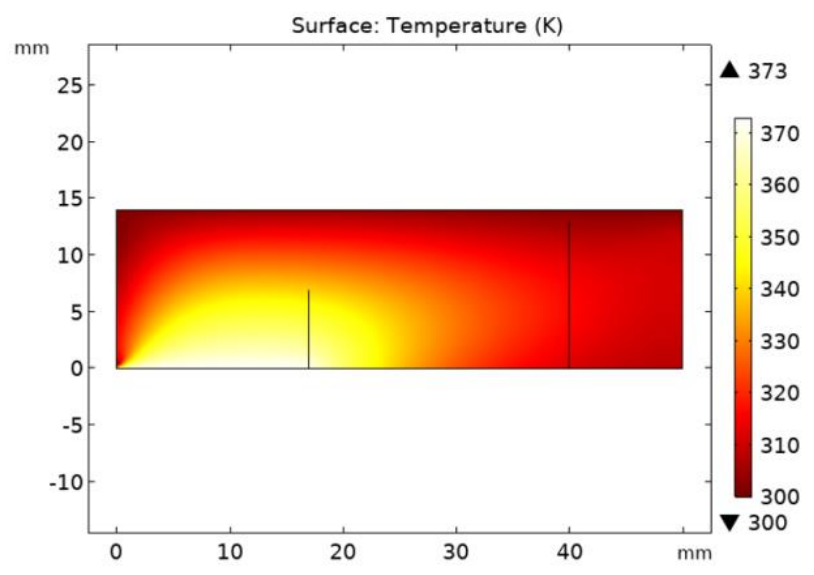

Fig. 5 - Temperature gradient surface plot showing also how the flow above the heater affects its profile. 


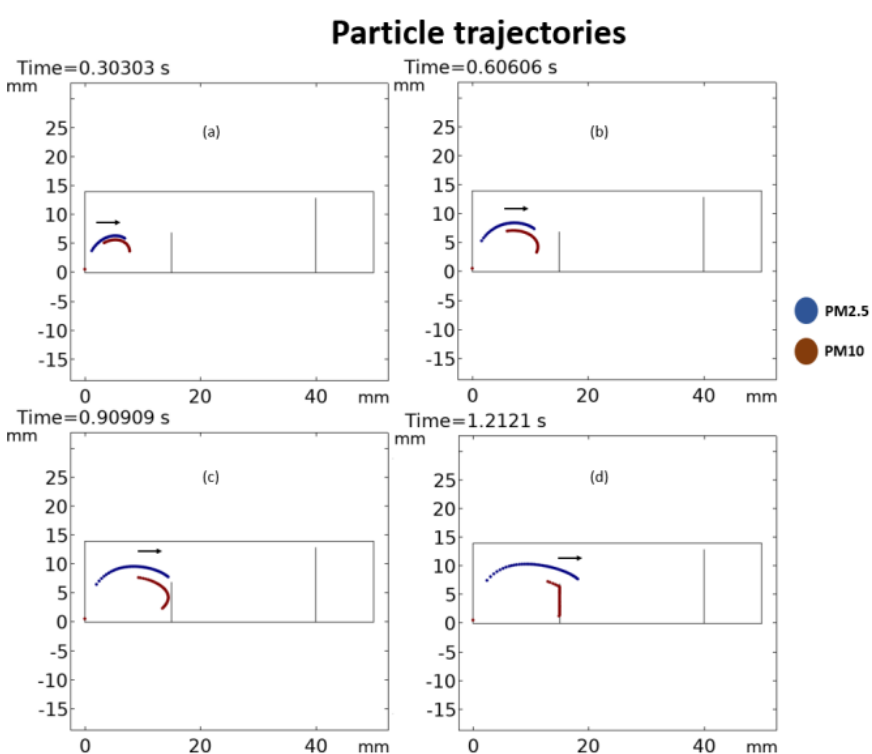

Fig. 6 - PM deposition simulation results snapshots showing particle flow trajectories in the sensing chamber including size discrimination via thermophoresis according to different sizes for the times (a) 0.30303 secs (b) 0.60606 secs (c) 0.90909 secs (d) 1.2121 secs.

\section{Particle trajectories and deposition}

Particle trajectories obtained by coupling the results of the Non-Isothermal flow with particle tracing module in a parametric sweep for different time steps are shown in Fig. 6 and Fig. 7. This is a combined stream of PM10 and PM2.5 which enter the sensing chamber simultaneously flow over the heater. In this case, the particles are similar in all chemical and physical properties apart from size and mass, and thus, respond differently to thermophoretic stimulation. The stream separates into two and are deposited on two different surfaces (since smaller particles are pushed farther away from the heater surface by the thermophoretic force than larger and heavier particles). This method can be used to collect multiple particle sizes (more than the 2 shown here) and produce statistically relevant information about each size range. Here, 50 particles of each size were released, and all were successfully deposited on the desired electrode surfaces as shown. The presented particle trajectories are snapshots in time from the simulation results of the time-dependent study. An animation of all the snapshots across all time steps is presented in the supplementary material.

\section{Capacitive detection}

Fig. 8 shows a comparison of the capacitive jumps for sensors with different electrode widths $(L=1 \mu \mathrm{m}$ and $L=25 \mu \mathrm{m})$ at different heights above the electrode surface for the same inter-electrode spacing $(g=1 \mu \mathrm{m})$. Both plots represent the particle movement vertically away from the surface of the electrodes. The strongest signal was obtained closest to the surface ( $3.5 \mu \mathrm{m}$ above the surface) in all cases. As shown in the plots, the signal level increases across panels from $1 \mu \mathrm{m}$ electrode width to $25 \mu \mathrm{m}$ electrode width with $1 \mu \mathrm{m}$ interelectrode spacing.

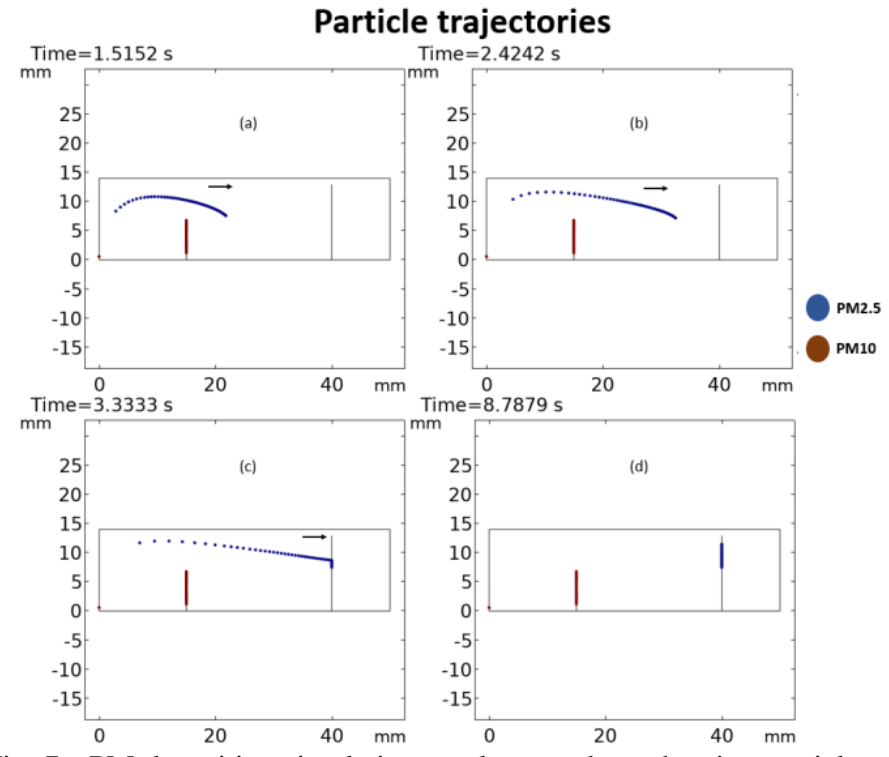

Fig. 7 - PM deposition simulation results snapshots showing particle flow trajectories in the sensing chamber including size discrimination via thermophoresis according to different sizes for the times (a) 1.5152 secs (b) 2.4242 secs (c) 3.3333 secs (d) 8.7879 secs.

Also, there is a noticeable reduction in the capacitive change as the height above the electrode is increased. However less noticeable is the effect of the dielectric constant $(\varepsilon)$. As it increases, the benefit in signal level also shrinks by so much that it is not a huge benefit beyond $\varepsilon=7$, and as the particle moves farther away from the electrode surface, it becomes even more insignificant across both plots. This result is confirmed by investigating the capacitance jumps for two different positions at for different/increasing for two electrode widths particle relative permittivity's presented in Fig. 9.

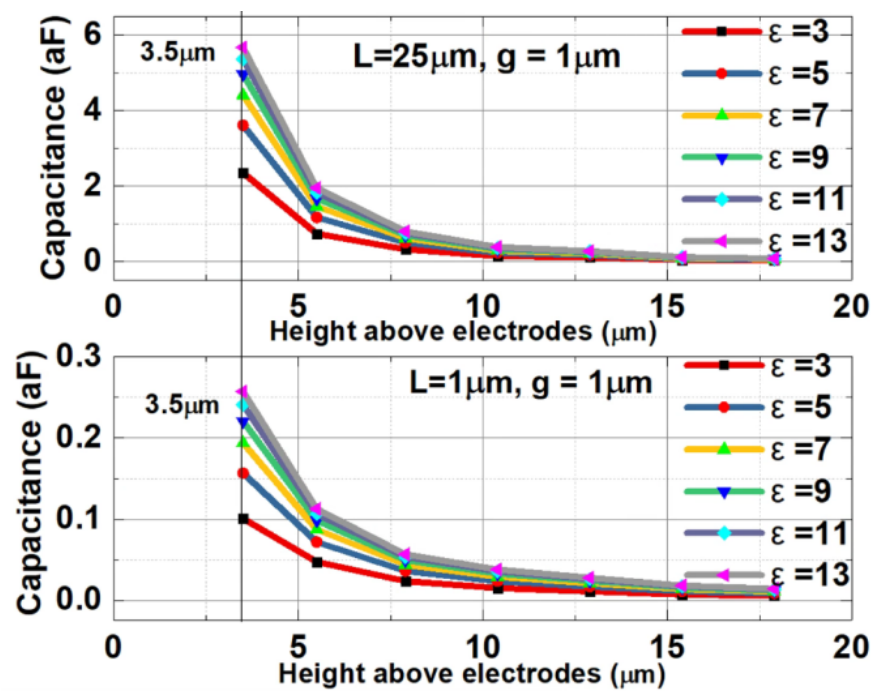

Fig. 8 - Electrostatic results showing the capacitive jumps detected for a single particle with different dielectric constants at different heights above the electrodes but the same inter-electrode spacing. The particle is located midway between the electrode fingers. The signal amplitude is increased by an order of magnitude as the electrode width is increased from $1 \mu \mathrm{m}$ to $25 \mu \mathrm{m}$. 

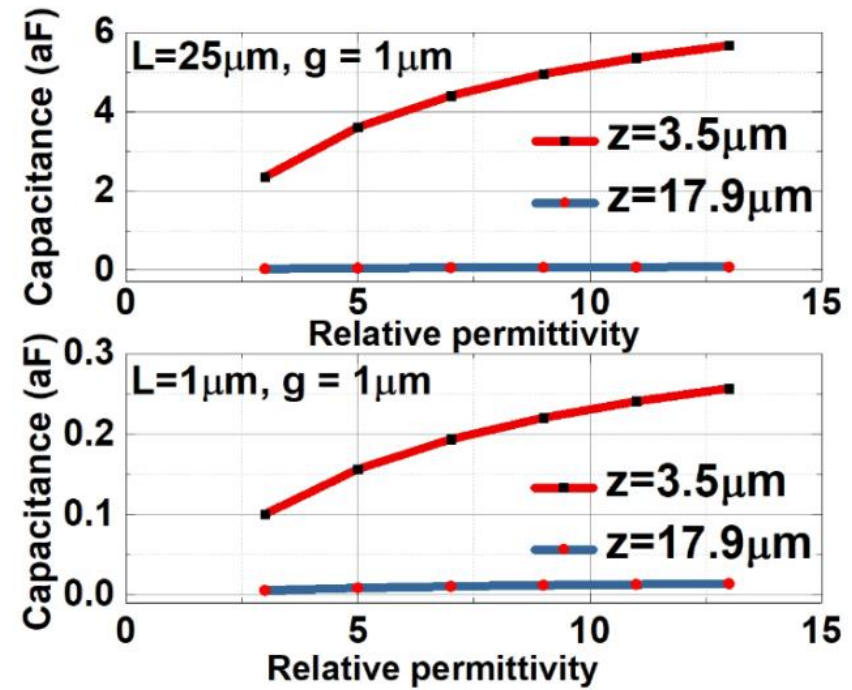

Fig. 9 - Electrostatic results showing the capacitive jumps detected for a single particle with different dielectric constants at particle heights $z=3.5 \mu \mathrm{m}$ and $z=17.9 \mu \mathrm{m}$ above the electrode surface showing that the effect of increasing dielectric constant diminishes as the particle moves away from the electrode surface.

At $z=3.5 \mu \mathrm{m}$, there is a huge gain (> 100\%) by increasing dielectric constant from 3 to 13 as shown, although, there is a gradual reduction in the increase from step to step. The influence of dielectric constant is maximum on the electrode surface, and almost negligible at around $17 \mu \mathrm{m}$ above it as shown for $z=17.9 \mu \mathrm{m}$. This discussion has not yet considered the influence of the inter-electrode spacing, especially as the electrode width increases.

Fig. 10 shows the results for this simulation where for each segment, each line represents the measured capacitance jumps caused by single particles of the same spacing but different electrode widths. Here the inter-relationship between the parameters becomes obvious. As previously mentioned, the expectation is for the capacitance to increase as the electrode width increases, increase as the dielectric constant increases and decrease as the inter-electrode spacing increases. However, going from $g=1 \mu \mathrm{m}$ to $g=10 \mu \mathrm{m}$, the values of the electrode width $L$ producing the largest changes in the capacitance value vary as follows. For the smallest inter-electrode gap $(g=1$ $\mu \mathrm{m})$, largest capacitance changes correspond to the largest electrode width $(L=25 \mu \mathrm{m})$. For $g=3 \mu \mathrm{m}$, this occurs for $L=20 \mu \mathrm{m}$; while for $g=5 \mu \mathrm{m}, L=10 \mu \mathrm{m}$ sensor produces the largest capacitance changes and for $g=10 \mu \mathrm{m}$, the largest capacitance changes are shown by the sensor with $L=20 \mu \mathrm{m}$ as reported in Fig. 10. In all cases tested, $L=1 \mu \mathrm{m}$ gave the smallest value of capacitance changes, while $g=1 \mu \mathrm{m}$ results in the ideal inter-electrode spacing, whereas the thinnest electrodes have the smallest signal amplitude that may be difficult to detect. Thus, the optimal electrode width is not at either of these extremes.

A horizontal sweep of the sensor to probe for capacitance changes as the particle moves from one electrode finger to the other was carried out to identify the optimal electrode width. The results of this analysis are presented in Fig. 11.
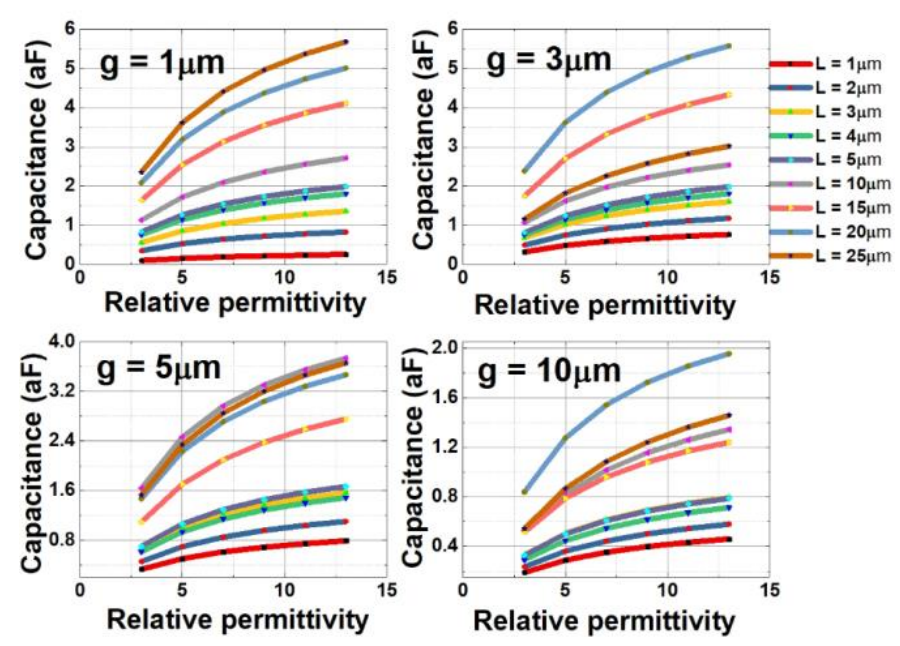

Fig. 10 - Results showing capacitance jumps as a function of interelectrode spacing, $g$, and electrode width. In general, for the same inter-electrode spacing, as electrode width increases, the capacitance change increases.

The ideal electrode width will in addition to having a good signal level also have a small difference between the largest and smallest signals. In Fig. $11, L=10 \mu \mathrm{m}, g=1 \mu \mathrm{m}$ is the optimal parameter combination since the difference between the lowest and the highest signal is $\sim 0.2 \mathrm{aF}$, smaller than $\sim 0.6 \mathrm{aF}$ for $L=$ $15 \mu \mathrm{m}$, and similar to $L=1 \mu \mathrm{m}$, and $L=5 \mu \mathrm{m}$, that have smaller signal levels. While this statement may seem contradictory at first the apparent contradiction is resolved when one considers that, for a coplanar arrangement of electrodes, the electric field is strongest at the edges and weakest at the centre of any electrode finger. The implication of this for particulate matter detection is that the signal level depends on where on the electrode the particle is deposited. With $g=1 \mu \mathrm{m}, L=25 \mu \mathrm{m}$ will give the highest signal but also more 'blind spots' in the middle, as shown in Fig 11.

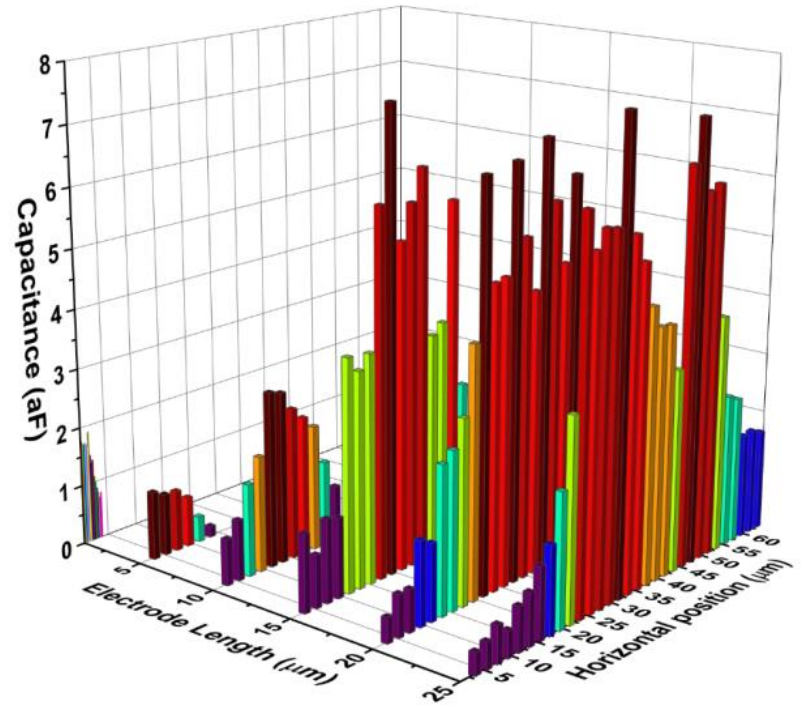

Fig. 11 - Electrostatic results showing the capacitive jumps detected for a single particle at different horizontal positions at different electrode widths (a) $L=1$, (b) $L=5$, (c) $L=10$, (d) $L=15$ but the same inter-electrode spacing, $g=1$. 


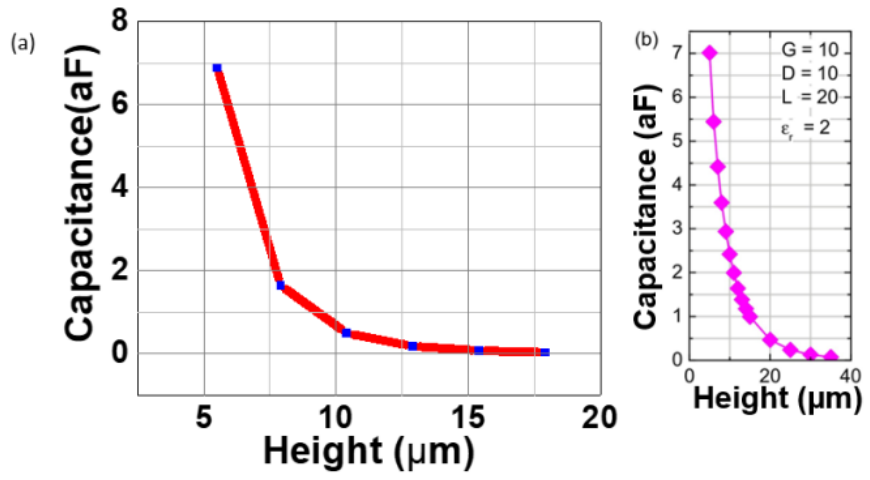

Fig. 12 - Comparison of the results for change in capacitance as a function of particle height above the electrode surface for (a) this work with (b) Carminati et al. [23], reproduced with permission.
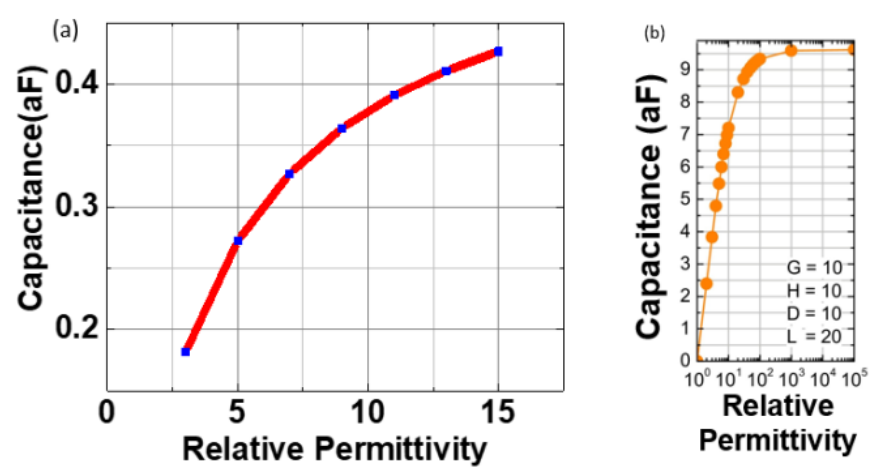

Fig. 13 - Comparison of the results for change in capacitance as a function of dielectric constant for (a) this work with (b) Carminati et al. [23] reproduced with permission.

However, an array of sensors monitoring the desired range would be needed to reduce the size range being collected in each of them thus allowing for more accuracy in the data collected.

\section{E. Comparison with literature}

The results of our simulations were compared with Carminati et al. [23] obtained for PM10.

Direct comparisons of capacitance measured with varying dielectric constant, and particle height above electrode, show good agreement as shown in Fig. 12 and Fig. 13.

\section{F. Extension of model to different particle types}

The model was extended, and simulation carried out for different kinds of particles that cover a large spectrum of the particulate matter constituents. Particles simulated include Silicon dioxide, Aluminum (III) oxide, Iron (II) oxide, Iron (III) oxide, Calcium oxide, Calcium carbonate, Magnesium carbonate, and sodium chloride. These particles all differ in density and relative permittivity. Both PM2.5 and PM10 for each were simulated to verify the particle discrimination presented in this work. The result showed that PM2.5 could still be detected with very high accuracy (>95\%) in spite of the different particle properties, however, the particle density proved to have a very significant impact on the deposition of PM10. Hence, even though two collection electrodes were used, the accuracy was less than that presented above.

Figure 14 shows the particle trajectory results.

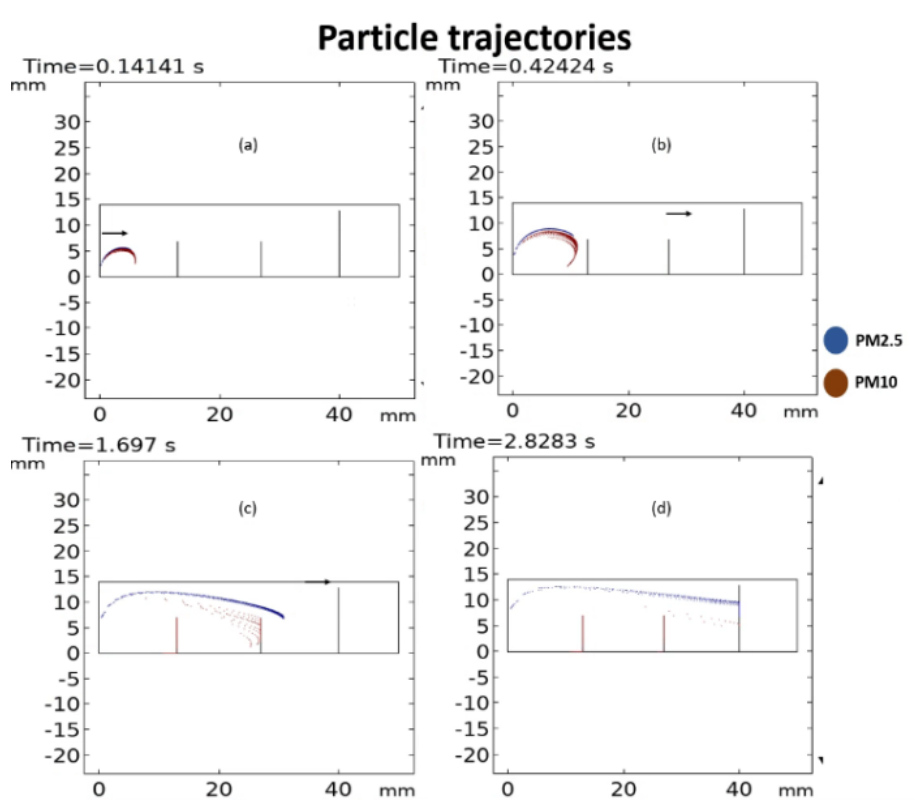

Fig. 14 - particle trajectory for multi-particle, multi-size simulation involving 8 different particle types. The result shows very high accuracy selective deposition of PM2.5 and lower accuracy for PM10. An animation of this is provided as supplementary information.

\section{G. Model limitations}

The limitations of this model include the assumption of a laminar flow regime, as well as an elastic collision at the chamber walls. Finally, the simulation model was tested with 8 types of particles with different physical and chemical properties. Our aim was to provide statistically-relevant information on the relative concentration of particles in different size ranges entrained in a sampled volume of airflow through a combined thermophoresis stimulation and capacitive detection method. At this stage the signal amplitude corresponding to the small variations in the capacitance for a single particle entering the active region of the sensor are in the sub-attofarad down to the zeptofarad (10-18 F - 10-21 F) range, which is beyond the resolution limits of many readout electronics because of parasitic elements. Also, given the vertical orientation of the sensor electrodes, it is not possible in this case to adopt a gravimetric mass measurement approach, as reported in some other works. Nevertheless, the results obtained in simulation and presented here are fully consistent with the model adopted and with studies reported in literature. The authors are confident that the approach proposed is promising towards a miniaturized "system-in-package" version of the device where parasitic capacitances are eliminated and the front-end electronics is capable of reading out the small capacitance values corresponding to particle deposition. Clogging on the sensor module can be done by cleaning and blow-drying. A bespoke pulsed flow can be used for particle collection. The sensor units may also be regularly replaced. 


\section{CONCLUSION}

This work has presented a capacitive PM2.5 sensor device targeting personalized exposure monitoring applications. The design and modelling performed showed that the device has sensitivity to single particles of the PM2.5 range with capacitive jump in excess of $0.48 \mathrm{zF} /$ particle even at $18 \mu \mathrm{m}$ above the electrode surface. The flow calculations and particle trajectories were obtained using COMSOL finite element analysis tool for laminar non-isothermal flow conditions. Thermophoresis was used to separate particles of PM10 and PM2.5 size range. Variations in sensor performance was shown as a function of electrode width, inter-electrode spacing, height above the electrodes and dielectric constant. Inter-relationship between these was also studied and optimal design parameters presented. Further development of this technology will enable pervasive citizen studies using non-intrusive sensor systems.

\section{APPENDIX}

Equations (A1) to (A4) define the forces acting on the particle.

$$
\begin{aligned}
& \boldsymbol{F}_{T}=-12 \pi r \frac{\mu^{2}}{\rho T} C_{F}\left(\frac{K}{(2 K+1)}\right) \nabla T \\
& K=\frac{k_{f}}{k_{s}} \\
& \boldsymbol{F}_{g}=m \boldsymbol{g} \\
& \boldsymbol{F}_{D}=6 \pi \mu r \boldsymbol{u}
\end{aligned}
$$

Where $k_{f}$ is the thermal conductivity of gas surrounding particle, $k_{s}$ is the thermal conductivity of particle, $K$ is a coefficient which depends on the flow regime usually as a function of $k_{s}$ and $k_{f}$.

Equations (A5) - (A7) define the electric displacement, electric field and the capacitance.

$$
\begin{aligned}
& \nabla \cdot D=\rho_{v} \\
& E=-\nabla V \\
& Q=C V
\end{aligned}
$$

Where $D$ is the electric displacement.

All other terms retain their usual meanings.

\section{ACKNOWLEDGMENT}

The authors would like to thank InnovateUK (GraphClean G.A. No. 71476-481865 and MP-SENS 73047-501268), EPSRC Sensors CDT (G.A. No. EP/KO3099X/1) and EPSRC CIMLAE (G.A. No. EP/LO15889/1) for their support in funding.

The authors would also like to thank the Federal Government of Nigeria for the funding for Pelumi's studies through the Presidential Special Scholarship for Innovation and Development (PRESSID) managed by the National Universities Commission (NUC) and funded by the Petroleum Technology Development Fund (PTDF). The authors would like to thank Dr. Varindra Kumar for reviewing the article before its submission.

\section{REFERENCES}

[1] N. Hodas, Q. Meng, M. M. Lunden, and B. J. Turpin, "Toward refined estimates of ambient PM2.5 exposure: Evaluation of a physical outdoor-to-indoor transport model," Atmos. Environ., vol. 83, pp. 229-236, 2014.

[2] N. Li, M. Hao, R. F. Phalen, W. C. Hinds, and A. E. Nel, "Particulate air pollutants and asthma: A paradigm for the role of oxidative stress in PM-induced adverse health effects," Clin. Immunol., 2003.

[3] A. C. Pope, R. T. Burnett, D. Krewski, M. Jerrett, Y. Shi, E. E. Calle, and M. J. Thun, "Cardiovascular mortality and exposure to airborne fine particulate matter and cigarette smoke shape of the exposure-response relationship," Circulation, 2009.

[4] X. Wu, R. Basu, B. Malig, R. Broadwin, K. Ebisu, E. B. Gold, L. Qi, C. Derby, and R. S. Green, "Association between gaseous air pollutants and inflammatory, hemostatic and lipid markers in a cohort of midlife women," Environ. Int., vol. 107, pp. 131-139, 2017.

[5] A. D. Kappos, P. Bruckmann, T. Eikmann, N. Englert, U. Heinrich, P. H^ppe, E. Koch, G. H. M. Krause, W. G. Kreyling, K. Rauchfuss, P. Rombout, V. Schulz-Klemp, W. R. Thiel, H.-E. Wichmann, and A. Kappos, "Health effects of particles in ambient air," Int. J. Hyg. Environ. Heal., vol. 207, pp. 399-407, 2004.

[6] L. Samek, Z. Stegowski, L. Furman, K. Styszko, K. Szramowiat, and J. Fiedor, "Quantitative Assessment of PM 2.5 Sources and Their Seasonal Variation in Krakow," Water Air Soil Pollut., vol. 228, 2017.

[7] Y.-H. Lim, H.-J. Bae, S.-M. Yi, E. Park, B.-E. Lee, and Y.-C. Hong, "Vascular and cardiac autonomic function and PM 2.5 constituents among the elderly: A longitudinal study," Sci. Total Environ., vol. 607-608, pp. 847-854, 2017.

[8] S.-T. Liu, C.-Y. Liao, and H.-W. Kuo, "The Effects of PM2.5 from Asian Dust Storms on Emergency Room Visits for Cardiovascular and Respiratory Diseases," Int J Env. Res Public Heal., vol. 14, no. 4, 2017.

[9] Y. Ma, Y. Zhao, S. Yang, J. Zhou, J. Xin, S. Wang, and D. Yang, "Short-term effects of ambient air pollution on emergency room admissions due to cardiovascular causes in Beijing, China," Environ. Pollut., vol. 230, pp. 974-980, 2017.

[10] J.-H. Chang, S.-C. Hsu, K.-J. Bai, S.-K. Huang, and C.-W. $\mathrm{Hsu}$, "Association of time-serial changes in ambient particulate matters (PMs) with respiratory emergency cases in Taipei's Wenshan District," PLoS One, vol. 12, no. 7, 2017.

[11] R. Liu, J. Zeng, X. Jiang, J. Chen, X. Gao, L. Zhang, and T. $\mathrm{Li}$, "The relationship between airborne fine particle matter and emergency ambulance dispatches in a southwestern city in Chengdu, China," Environ. Pollut., vol. 229, pp. 661-667, 2017.

[12] N. Thi, T. Nhung, H. Amini, C. Schindler, M. K. Joss, T. M. Dien, N. Probst-Hensch, L. Perez, and N. Künzli, "Short-term association between ambient air pollution and pneumonia in children: A systematic review and meta-analysis of timeseries and case-crossover studies," Environ. Pollut., vol. 230, pp. 1000-1008, 2017.

[13] J. O. Anderson and J. G. Thundiyil, "Clearing the Air: A Review of the Effects of Particulate Matter Air Pollution on Human Health," J. Med. Toxicol., vol. 8, pp. 166-175, 2012.

[14] R. B. Husar, "Atmospheric Particulate Mass Monitoring with a Beta Radiation detector," Atmos. Environ., vol. 8, pp. 183188, 1974.

[15] E. S. Macias and R. B. Husar, "Atmospheric Particulate Mass Measurement with Beta Attenuation Mass Monitor," Environ. Sci. Technol., vol. 10, no. 9, pp. 904-907, 1976. 
[16] H. Patashnick and E. G. Rupprecht, "Continuous PM-10 Measurements Using the Tapered Element Oscillating Microbalance," J. Air Waste Manage. Assoc., vol. 41, no. 8, pp. 1079-1083, 1991.

[17] P. Kumar, L. Morawska, C. Martani, G. Biskos, M. Neophytou, S. Di Sabatino, M. Bell, L. Norford, and R. Britter, "The rise of low-cost sensing for managing air pollution in cities," Environ. Int., vol. 75, pp. 199-205, 2015.

[18] S. Steinle, S. Reis, C. E. Sabel, S. Semple, M. M. Twigg, C. F. Braban, S. R. Leeson, M. R. Heal, D. Harrison, C. Lin, and $\mathrm{H}$. Wu, "Personal exposure monitoring of PM2.5 in indoor and outdoor microenvironments," Sci. Total Environ., vol. 508, pp. 383-394, 2015.

[19] L. G. Occhipinti and P. W. Oluwasanya, "Particulate Matter Monitoring : Past, Present and Future," Int. J. Earth Environ. Sci., vol. 2, no. 144, pp. 2-5, 2017.

[20] X. Li, E. Iervolino, F. Santagata, J. Wei, C. A. Yuan, P. M. Sarro, and G. Q. Zhang, "Miniaturized particulate matter sensor for portable air quality monitoring devices," in Proceedings of IEEE Sensors, 2014, pp. 2151-2154.

[21] M. Budde, R. El Masri, T. Riedel, and M. Beigl, "Enabling low-cost particulate matter measurement for participatory sensing scenarios," in Proceedings of the 12th International Conference on Mobile and Ubiquitous Multimedia, 2013, pp. $1-10$.

[22] J. Sun, Z. Liu, K. Yang, and Y. Lu, "A miniature system for particulate matter (PM) measurement," in Proceedings of IEEE Sensors, 2015, pp. 1-4.
[23] M. Carminati, L. Pedalà, E. Bianchi, F. Nason, G. Dubini, L. Cortelezzi, G. Ferrari, and M. Sampietro, "Capacitive detection of micrometric airborne particulate matter for solidstate personal air quality monitors," Sens Act, A Phy., vol. 219, pp. 80-87, 2014.

[24] S. Iacopini and R. Piazza, "Thermophoresis in protein solutions," Europhys. Lett., vol. 63, no. 2, pp. 247-253, 2003.

[25] C. J. Wienken, P. Baaske, U. Rothbauer, D. Braun, and S. Duhr, "Protein-binding assays in biological liquids using microscale thermophoresis," Nat. Commun., vol. 1, no. 7, pp. $1-7,2010$.

[26] D. I. Fotiadis and K. F. Jensen, "Thermophoresis of solid particles in Horizontal Chemical Vapour Deposition reactors," J. Cryst. Growth, vol. 102, pp. 743-761, 1990.

[27] H. R. Jiang, N. Yoshinaga, and M. Sano, "Active motion of a Janus particle by self-thermophoresis in a defocused laser beam," Phys. Rev. Lett., vol. 105, no. 26, pp. 1-4, 2010.

[28] S. L. Goren, "Thermophoresis of aerosol particles in the laminar boundary layer on a flat plate," J. Colloid Interface Sci., 1977.

[29] W. L., Talbot; R., Cheng; R.W., Schefer; D.R., "Thermophoresis of particles in a Heated Boundary Layer," J. Fluid Mech., vol. 101, no. 4, pp. 737-758, 1980.

[30] W. Wang, W. Ma, Q. Wang, and H. Ren, "Conformal mapping for multiple terminals," Nat. Sci. Reports, vol. 6, no. 36918, 2016.

[31] J. R. Brock, "On the theory of thermal forces acting on aerosol particles," J. Colloid Sci., vol. 17, pp. 768-780, 1962.

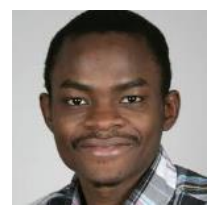

Mr Pelumi W. Oluwasanya is a $\mathrm{PhD}$ candidate in Engineering at Cambridge University. He has a BSc (Hons) in Electrical/Electronic Engineering from Olabisi Onabanjo University with First Class, a MSc in Signal processing and communications from The University of Edinburgh and a MRes in Sensor Technologies and Applications from the University of Cambridge. His research focus is on sensors; their design using new materials, fabrication and applications in air quality monitoring, wearables and healthcare.

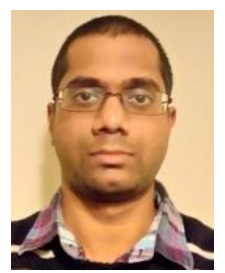

Dr Girish Rughoobur is at the Microsystems Technology Laboratories, Massachusetts Institute of Technology (MIT), USA as a Research Associate. He is currently working on improving the performance of Si field emission arrays in low vacuum for a wide range of applications. He has a MEng in Electrical and Electronic Engineering from the University College London (UCL), UK in 2012. Girish obtained a PhD in Engineering in 2017 from the University of Cambridge, UK for his work on the fabrication and characterization of in-liquid thin film bulk acoustic resonators for sensors/biosensors. Girish is also a member of the Institution of Engineering and Technology and the Institution of Electrical and Electronics Engineers.

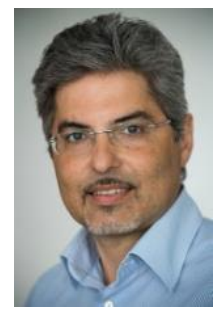

Dr Luigi G. Occhipinti is a Director of Research and Principal Investigator at Cambridge University Engineering Department of Cambridge University. He has developed science and innovation for more than 20 years in emerging technology areas for the post-CMOS roadmap, smart systems heterogeneous integration, polymer and printed electronics, advanced bio-systems and molecular diagnostics, advanced signal processing and nonlinear computation, advanced mechanical, optical and chemical sensor technologies, with focus on miniaturization and advanced packaging technologies for smart devices and products. He is the Deputy Director and Chief Operating Officer of the Cambridge Graphene Centre. 